\title{
MBD2 is a transcriptional repressor belonging to the MeCP1 histone deacetylase complex
}

\author{
Huck-Hui Ng${ }^{1}$, Yi Zhang ${ }^{2}$, Brian Hendrich ${ }^{1}$, Colin A. Johnson ${ }^{3}$, Bryan M. Turner ${ }^{3}$, \\ Hediye Erdjument-Bromage ${ }^{4}$, Paul Tempst ${ }^{4}$, Danny Reinberg ${ }^{2} \&$ Adrian Bird $^{1}$
}

Mammalian DNA is methylated at many CpG dinucleotides. The biological consequences of methylation are mediated by a family of methyl-CpG binding proteins ${ }^{1-4}$. The best characterized family member is MeCP2, a transcriptional repressor that recruits histone deacetylases ${ }^{5-7}$. Our report concerns MBD2, which can bind methylated DNA in vivo and in vitro ${ }^{4}$ and has been reported to actively demethylate DNA (ref. 8). As DNA methylation causes gene silencing, the MBD2 demethylase is a candidate transcriptional activator. Using specific antibodies, however, we find here that MBD2 in HeLa cells is associated with histone deacetylase (HDAC) in the MeCP1 repressor complex ${ }^{1,9}$. An affinity-purified HDAC1 corepressor complex ${ }^{10,11}$ also contains MBD2, suggesting that MeCP1 corresponds to a fraction of this complex. Exogenous MBD2 represses transcription in a transient assay, and repression can be relieved by the deacetylase inhibitor trichostatin A (TSA; ref. 12). In our hands, MBD2 does not demethylate DNA. Our data suggest that HeLa cells, which lack the known methylationdependent repressor MeCP2, use an alternative pathway involving MBD2 to silence methylated genes.

Previous work showed that Mbd2b, a truncated form of mouse Mbd2 (Fig. 1a), binds DNA in vitro in a methylation-dependent manner ${ }^{4}$. Full-length recombinant mouse MBD2a (Fig. 1a) also formed a complex with a densely methylated probe in a bandshift assay, but did not bind to non-methylated counterpart (Fig. 1b). To search for MBD2-associated proteins, we first raised polyclonal antisera against recombinant MBD2. Antibodies were tested on western blots of HeLa nuclear extract and assessed for their ability to discriminate between MBD2 (in particular the MBD3-like MBD2b form) and its relative, MBD3 (ref. 4). Antiserum S923 bound to recombinant MBD2b, but not to MBD3 (Fig. 1c). Antiserum R593 recognized MBD2b and cross-reacted with MBD3 (Fig. 1c). In HeLa extract, S923 antibodies detected an approxi- $a$

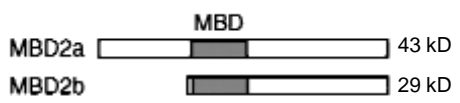

$d$

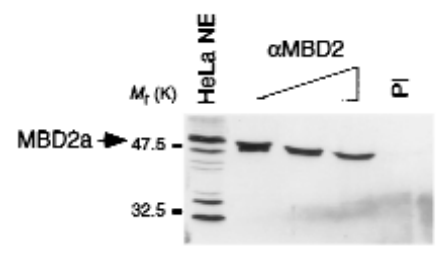

$b$

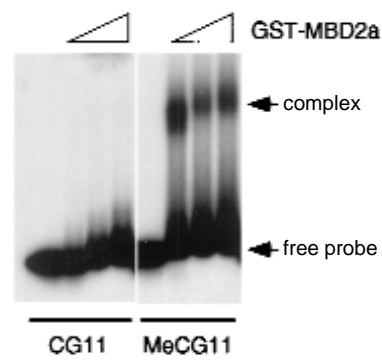

$e$

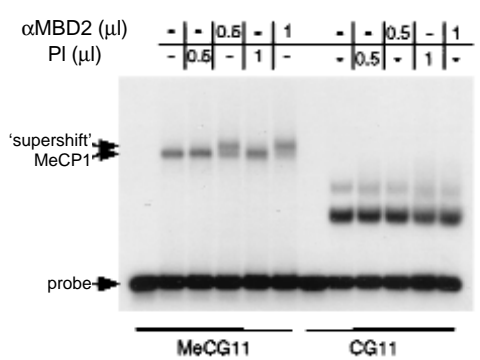

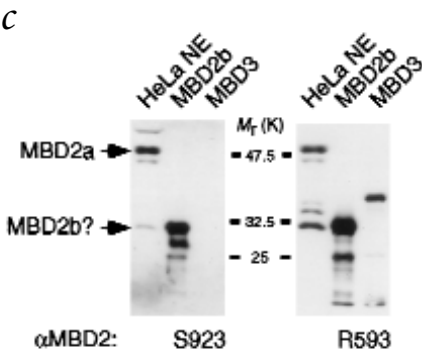

$f$

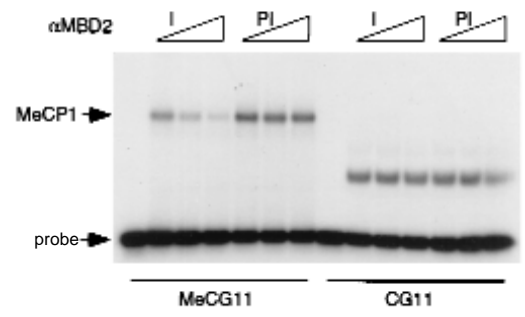

Fig. $1 \mathrm{MBD} 2 \mathrm{a}$ binds methylated DNA specifically and is part of the methyl-CpG binding complex, MeCP1. a, Maps of full-length MBD2a and a hypothetical truncated form, MBD2b, that initiates at a downstream methionine ${ }^{4}$. $\boldsymbol{b}$, Recombinant MBD2a forms a complex with probe MeCG11 (26 methyl-CpGs), but not with the non-methylated form CG11. c, Anti-MBD2 antisera S923 and R593 probed against western blots of HeLa nuclear extract (26 $\mu$ g protein; HeLa NE), recombinant MBD2b (30 ng; ref. 4) and recombinant MBD3 (30 ng; ref. 4). d, Immunoprecipitates with anti-MBD2 antiserum S923 ( $\alpha$ MBD2) contained only the 48-kD MBD2a protein. MBD2a always resolves as a doublet band for unknown reasons; the doublet resolves less well at higher concentrations of antibody due to the increased presence of closely migrating IgG. The control was pre-immune serum (PI). e, S923 anti-MBD2 antibodies supershift the bandshift complex between MeCP1 and the methylated DNA probe MeCG11. No supershift was seen with pre-immune serum or of the non-specific CG11 complex (PI). $\boldsymbol{f}$, Depletion of MBD2 from HeLa cell nuclear extracts with $\mathrm{S} 923$ antiserum $(2.5,5$ and $10 \mu \mathrm{l})$ causes loss of MeCP1 complex in a bandshift assay. Pre-immune serum (PI) has no effect.

${ }^{1}$ Institute of Cell and Molecular Biology, University of Edinburgh, The King's Buildings, Edinburgh EH9 3JR, UK. ${ }^{2}$ Howard Hughes Medical Institute, Division of Nucleic Acids Enzymology, Department of Biochemistry, University of Medicine and Dentistry of New Jersey, Robert Wood Johnson Medical School, Piscataway, New Jersey 08854, USA. ${ }^{3}$ Department of Anatomy, The Medical School, University of Birmingham, Birmingham B15 2TT, UK. ${ }^{4}$ Molecular Biology Program, Memorial Sloan Kettering Cancer Center, 1275 York Avenue, New York, New York 10021, USA. Correspondence should be addressed to A.B. (e-mail: A.Bird@ed.ac.uk). 
Fig. $2 \mathrm{MBD} 2$ is associated with histone deacetylases in the MeCP1 complex. a, Anti-MBD2 antibodies co-immunoprecipitate HDAC activity from HeLa cell extracts, but pre-immune serum (PI) and control antibodies against MBD1 and CDK7 do not. b,c, AntiHDAC1 antibodies $(10,20$ and $30 \mu \mathrm{l})$ deplete MeCP1 bandshift activity $(b)$ and MBD2 (c) from a HeLa nuclear extract. Control nonimmune serum does not deplete MeCP1 or MBD2. MBD2 was detected by western blot using $\$ 923$ antiserum. d, Anti-MBD2 antibodies co-immunoprecipitate HDAC1, HDAC2 and RbAp48 from HeLa nuclear extracts. Co-migration with immunoglobulin heavy chain leads to aberrant migration of RbAp48 in the immunoprecipitated lane. e, Polypeptides retained on HDAC1 ( $\alpha$ HDAC1) and GST ( $\alpha$ GST) antibody columns after separation by SDS-PAGE followed by silver staining. Known components of the complex ${ }^{11}$ are labelled (right). Mass spectrometric and sequence analysis of the 43-kD band from the fraction isolated using anti-HDAC1 antibodies identified a peptide (VLYIDIDIHHGDGVEEAFYTTDR) contained in HDAC1, as well as a peptide (GLQGVGPGSNDETLLSAVASALHTSSAPITGQVSAAVEK) that matches the MBD2 sequence. It is not known whether HDAC1* represents an alternative form or degradation product of HDAC1. The data suggest roughly equimolar amounts of HDAC1* and MBD2 in this band (data not shown). $f$, MBD2a, RbAp46, RbAp48 and HDAC1 were detected by western blot in nuclear extract (input) and in complexes that were affinity purified with anti-HDAC1 antibodies.

mately 49-kD band corresponding to full-length MBD2a (in vitro-translated MBD2a has an identical mobility; data not shown), plus an approximately $30-\mathrm{kD}$ band that may be MBD2b or a degradation product. S923 does not cross-react with MeCP2, MBD1 or MBD4 (data not shown). We further tested the specificity of the S923 antiserum against soluble nuclear proteins by probing western blots of $\$ 923$ immunoprecipitates with the less-specific R593 antiserum. R593 recognizes MBD3 (Fig. 1c), but only MBD2a was detected in the precipitated material (Fig. $1 d$ ).

We used the highly specific S923 antiserum to determine whether MBD2 is a component of the methylCpG binding activity (MeCP1; ref. 1) previously implicated as a transcriptional repressor ${ }^{9,13}$. S923 antibodies were able to quantitatively supershift the MeCP1 complex with a probe methylated at multiple sites, whereas pre-immune serum was inert (Fig. 1e, MeCG11 lanes). A non-specific complex with the non-methylated probe ${ }^{9}$ was unaffected by either immune or pre-immune sera (Fig. 1e, CG11 lanes). When we used anti-MBD2 antibodies to deplete MBD2 from the HeLa extract, the MeCP1 complex was correspondingly depleted (Fig. 1f). Pre-immune serum did not deplete the MeCP1 complex, and neither antiserum affected the nonspecific complex with non-methylated DNA. We conclude that MBD2 is a component of the MeCP1 complex.

Several studies have indicated that methylation-dependent transcriptional repression involves deacetylation $6,7,14-17$. As $\mathrm{MeCP} 1$ is a likely repressor of methylated genes ${ }^{9}$, we asked whether its constituent MBD2 is associated with deacetylases. We found that S923 immunoprecipitates contained up to $27 \%$ of the total histone deacetylase activity of HeLa extracts (Fig. 2a). Moreover, anti-HDAC1 antibodies efficiently immunodepleted both MeCP1 (Fig. 2b) and MBD2 (Fig. 2c) from the extract, whereas control non-immune serum had no effect. In addition, several known protein components of corepressor complexes were co-immunoprecipitated by MBD2 antibodies from a HeLa extract (Fig. 2d), indicating that MeCP1 activity is due to a complex between MBD2, deacetylases HDAC1 and HDAC2, and RbAp48. As a negative control, we found that retinoblastoma protein, which associates with HDACs (refs 18-20), was not immunoprecipitated by anti-MBD2 (data not shown). Mass fingerprinting analysis of proteins affinity-purified using an anti-

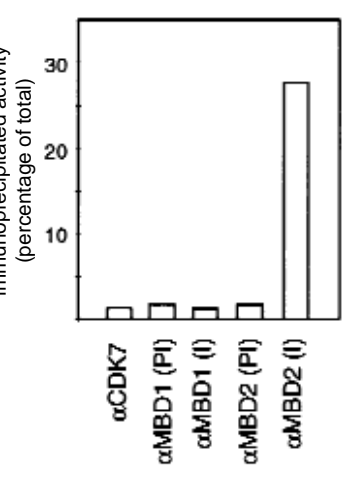

$b$

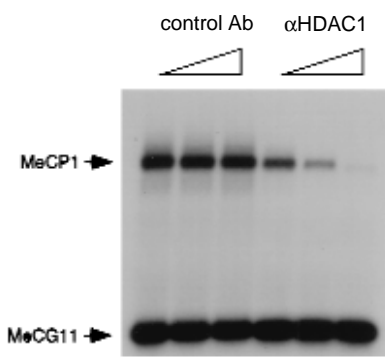

$d$

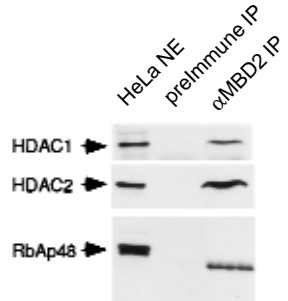

$f$

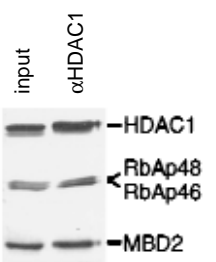

HDAC1 antibody $^{11}$ (Fig. 2e) indicated that MBD2 is a component of a corepressor complex. Western-blot analysis confirmed that MBD2 is present in complexes affinity purified with HDAC1 antibodies, together with $\mathrm{HDAC} 1 / 2$-associated proteins RbAp46 and RbAp48 (Fig. 2f).

A previous report claimed that a related methyl-CpG binding protein, MBD1, is a component of MeCP1 (ref. 3). Subsequent experiments with antibodies highly specific for MBD1 failed to supershift or immunodeplete MeCP1 (H.-H.N. and A.B., unpublished data) or to immunoprecipitate deacetylase activity (Fig. 2a). These and other results lead us to suspect that the anti-MBD1 antibody used in the previous study may have cross-reacted with MBD proteins that were unknown at the time. Recent work confirms the proposal $^{3}$ that MBD1 is a methylation-dependent transcriptional repressor, but argues against its involvement in MeCP1 of HeLa cells (H.-H.N. and A.B., unpublished data).

HeLa cells lack detectable amounts of the known methylationdependent repressor MeCP2 (Fig. 3a), but are nevertheless able to repress transfected methylated genes ${ }^{9}$. We initially determined whether repression in the effective absence of MeCP2 was HDAC dependent by transiently transfecting a luciferase reporter gene that contained a cluster of 20 methylatable HhaI sites (CG11) immediately upstream of the SV40 promoter (Fig. $3 b, c$ ). The M.HhaI-methylated reporter construct was transcribed at approximately $30 \%$ of the level seen in non-methylated controls (Fig. $3 b$ ). The same construct lacking the CG11 cluster (originally used to 
Fig. 3 MBD2 can account for deacetylase-dependent repression of methylated genes in HeLa cells. a, Western blots show that MeCP2 is not detectable in HeLa extracts ( $26 \mu \mathrm{g})$, but is abundant in rat brain extracts $(20 \mu \mathrm{g})$. Controls show that $\mathrm{mSin} 3 \mathrm{~A}$ is present in both extracts. $\boldsymbol{b}$, Transcription of the transiently transfected CG11-pGL2 construct (c) is repressed by methylation of Hhal sites (Hhal M+), but not by mock methylation (mock M-). Repression is relieved by $100 \mathrm{ng} / \mathrm{ml}$ TSA. Per cent relative activity of the promoters is expressed as the ratio of luciferase to $\beta$-galactosidase activities. c, Map of reporter gene constructs. CG11-pGL2 contains 45 methylatable Hhal sites, including the promoter-proximal CG11 cluster. Comparison of DNA polymerase- $\beta$ and $\beta$-actin promoter constructs, each with five Gal4 binding sites. The SV40 enhancer is marked (Enh). d,e, Co-transfection of a construct encoding MBD2a fused to the Gal4 DNA-binding domain together with the DNA pol $\beta(d)$ or $\beta$-actin (e) reporters into mouse $L$ cells causes transcriptional repression. Relative activities are the ratios of luciferase to $\beta$-galactosidase (d) or $\beta$-galactosidase to luciferase $(e)$ activities. TSA $(100 \mathrm{ng} / \mathrm{ml})$ relieves repression of the DNA pol $\beta$ reporter.

identify MeCP1 activity $^{1}$ ) was minimally repressed by HhaI site methylation (X. Nan and A.B., unpublished data). TSA treatment reduced methylationdependent repression (Fig. $3 b$ ), indicating that deacetylation is involved. We next asked whether MBD2 was itself a repressor by transiently expressing MBD2a fused to the Gal4 DNA-binding domain. Transcription of a co-transfected reporter gene encoding the human DNA polymerase- $\beta$ promoter (plus Gal4 DNA-binding sites) was inhibited by Gal4-MBD2a, but repression was abolished by TSA (Fig. $3 d$ ). A reporter under control of the human $A C T B$ (encoding $\beta$-actin) promoter was also repressed by the fusion protein, but repression was weakly reduced by TSA (Fig. 3e). Promoterspecific responses to derepression by TSA have been observed ${ }^{20}$ previously. Repression of both promoters depended on the presence of Gal4-binding sites in the reporter construct (data not shown). The results indicate that repression of at least some promoters by MBD2 depends on deacetylation.

Given the unexpected association of a putative demethylase with transcriptional repression complexes, we tested the demethylation activity of in vitro-translated mouse $\mathrm{e}^{4}$ or human ${ }^{8}$ MBD2b expression constructs. Translation products complexed with methylated $a$

$\mathcal{C}$

$d$

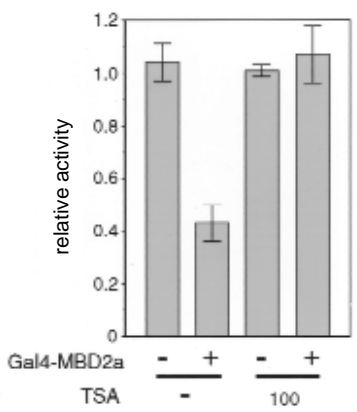

$e$

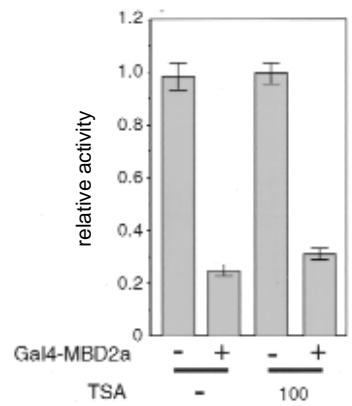

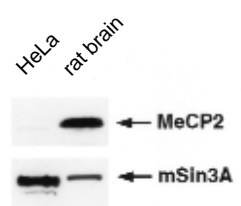

$b$

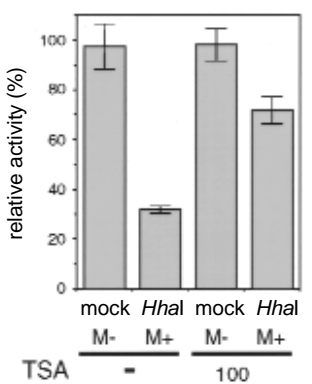

CG11-pGL2
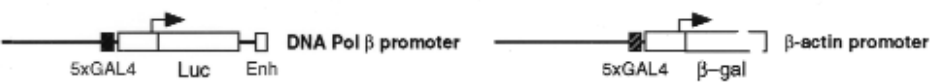

5XGAL4 $\beta$-gal

DNA (Fig. 4a), indicating correct protein folding, but methylated CCGG and GCGC sites in the same probe (Fig. 4c) were not susceptible to HpaII or HhaI after prolonged incubation under published conditions ${ }^{8}$ (Fig. 4b). Moreover, we did not observe dissociation of the MBD2 bandshift complexes due to demethyla-

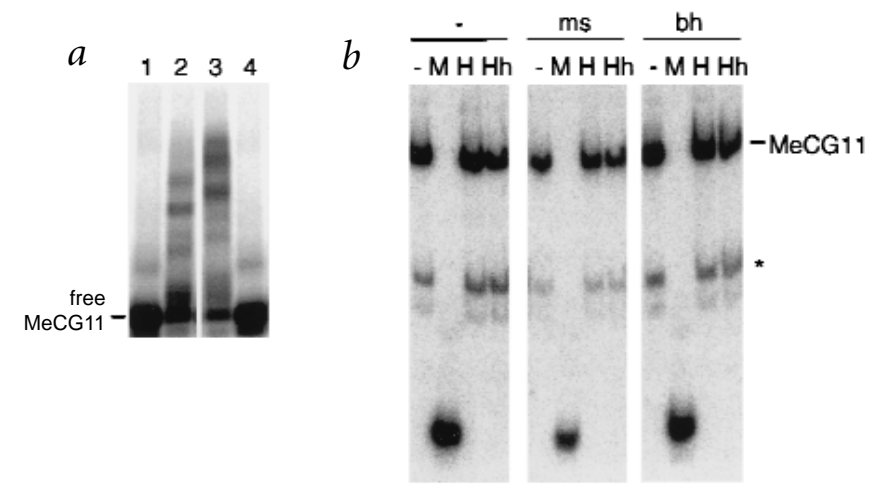

C AA'IILGAGCIEGGIACCCGTACCCAAGMGMMGCCTGGMGCMGGGCMGGCTCCAAGMGMMGCCTGGMCCMGGCAAGM

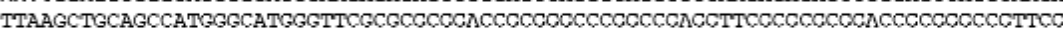

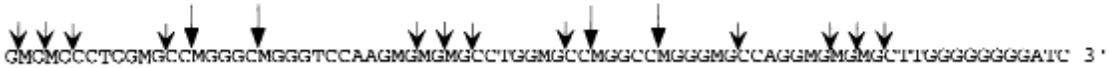
CGCGCGGACCGCGGGCCGGCCCAGGTCGCGCGCGGACCCGGGCGGGCCGCGGTCCGCGCGCGAACCCCCCCCTAG 5 , 
tion of binding sites during the prolonged assay (Figs $1 b$ and $4 a$ ). Attempts to demethylate other oligonucleotide and plasmid substrates with bacterially expressed $\mathrm{MBD} 2$, in vitro-translated MBD2b or anti-MBD2 immunoprecipitates from HeLa extracts were similarly unsuccessful (data not shown). It may be that MBD2 has a dual role as both a DNA methylation-dependent repressor and an activator of genes that are silenced by methylation. Diametrically opposed activities might theoretically be combined in a protein that serves as a switch between active and inactive chromatin states. This speculative hypothesis depends, however, on verification of the demethylase activity of MBD2.

Our data uncover a novel mediator of methylated gene silencing. Although MeCP2 and MBD2 both repress transcription of methylated DNA via a deacetylase-containing corepressor complex, there are differences between them. Tightly bound MeCP2 (refs 2,21) has the character of a structural component of the chromosome that ensures long-term silencing of methylated sequences. $\mathrm{MBD} 2 / \mathrm{MeCP} 1$, on the other hand, is released from nuclei by low salt $^{1}$, suggesting that it is not stably complexed with DNA. The MeCP1 subset of the HDAC corepressor complex may be a catalytic complex that alters methylated DNA-containing chromatin during a transient visit. There is also a difference in binding specificity between $\mathrm{MeCP} 1$ and MeCP2. MeCP2 binds to single methylated sites ${ }^{22}$, whereas $\mathrm{MeCP} 1$ requires regions of dense $\mathrm{CpG}$ methylation ${ }^{1}$. Conceivably, each MeCP1 complex contains multiple molecules of MBD2, thereby enhancing binding to local clusters of methyl-CpG. Assessment of this possibility awaits future purification and compositional analysis of $\mathrm{MeCP} 1$. Already, the differences between MBD2 and MeCP2 suggest that they may have somewhat different roles in transcriptional silencing.

\section{Methods}

Plasmids. We constructed plasmid pCMV-Gal4-MBD2a by inserting a $\mathrm{NaeI} / \mathrm{SspI}$ fragment of MBD2a cDNA (ref. 4) into BamHI-blunted pCMVGal4 vector ${ }^{5}$ and pGEX5-MBD2a by inserting the same NaeI/SspI fragment of MBD2a cDNA into BamHI-blunted pGEX5x1 vector (gift from S. MacNeill). We sequenced all constructs to confirm identity. CG11-pGL2 luciferase reporter was a gift from X. Nan (unpublished data). Plasmid pHis-dMTase was a gift from M. Szyf ${ }^{8}$. The Gal4-DNA polymerase- $\beta$ luciferase reporter ${ }^{23}$ was a gift from J. Millbrandt.

Transfection and reporter assays. We transfected mouse fibroblast $\mathrm{L}$ cells and $\beta$-galactosidase assays essentially as described ${ }^{5,6}$ and detected luciferase activity using a luciferase assay kit (Promega). Transfection mixes included reporter ( $2 \mu \mathrm{g}$; with Gal4-binding sites), effector ( $2 \mu \mathrm{g}$; Gal4MBD2a) and internal control reporter $(2 \mu \mathrm{g}$; either human ACTB promot-

1. Meehan, R.R., Lewis, J.D., McKay, S., Kleiner, E.L. \& Bird, A.P. Identification of a mammalian protein that binds specifically to DNA containing methylated CpGs. Cell 58, 499-507 (1989).

2. Lewis, J.D. et al. Purification, sequence and cellular localisation of a novel chromosomal protein that binds to methylated DNA. Cell 69, 905-914 (1992).

3. Cross, S.H., Meehan, R.R., Nan, X. \& Bird, A. A component of the transcriptional repressor MeCP1 is related to mammalian DNA methyltransferase and thrithorax-like protein. Nature Genet. 16, 256-259 (1997).

4. Hendrich, B. \& Bird, A. Identification and characterization of a family of mammalian methyl-CpG binding proteins. Mol. Cell. Biol. 18, 6538-6547 (1998).

5. Nan, X., Campoy, J. \& Bird, A. MeCP2 is a transcriptional repressor with abundant binding sites in genomic chromatin. Cell 88, 471-481 (1997).

6. Nan, X. et al. Transcriptional repression by the methyl-CpG-binding protein MeCP2 involves a histone deacetylase complex. Nature 393, 386-389 (1998).

7. Jones, P.L. et al. Methylated DNA and MeCP2 recruit histone deacetylase to repress transcription. Nature Genet. 19, 187-191 (1998).

8. Bhattacharya, S.K., Ramchandani, S., Cervoni, N. \& Szyf, M. A mammalian protein with specific demethylase activity for mCpG DNA. Nature 397, 579-583 (1999).

9. Boyes, J. \& Bird, A. DNA methylation inhibits transcription indirectly via a methyl-CpG binding protein. Cell 64, 1123-1134 (1991).

10. Zhang, Y., Iratni, R., Erdjument-Bromage, H., Tempst, P. \& Reinberg, D. Histone deacetylases and SAP18, a novel polypeptide, are components of a human $\operatorname{Sin} 3$ complex. Cell 89, 357-364 (1997).

11. Zhang, Y. et al. SAP30, a novel protein conserved between human and yeast, is a component of a histone deacetylase complex. Mol. Cell 1, 1021-1031 (1998).

12. Yoshida, M., Horinouchi, S. \& Beppu, T. Trichostatin A and trapoxin: novel chemical probes for the role of histone acetylation in chromatin structure and er driving $\beta$-galactosidase or pGL2 SV40 promoter/enhancer driving luciferase). We transfected HeLa cells with CG11-pGL2 luciferase reporter ( $1 \mu \mathrm{g}$; either mock methylated or methylated with $\mathrm{HhaI}$ methyltransferase) and human $\beta$-actin/ $\beta$-galactosidase reporter $(1 \mu \mathrm{g})$ using Lipofectamine (Gibco BRL) according to the manufacturer's instructions. We treated cells with TSA $(100 \mathrm{ng} / \mathrm{ml})$ for $24 \mathrm{~h}$ before collection.

Bandshifts, immunodepletion and immunoprecipitation assays. We performed bandshift assays as described ${ }^{3}$. For immunodepletion of MBD2 or MeCP1 activity, we immobilized the antibodies (5-30 $\mu \mathrm{l}$ serum) on protein-G sepharose (Pharmacia). After washing the beads with PBS (plus $1 \mathrm{M}$ $\mathrm{NaCl}$ and $0.1 \%$ Triton X-100) and binding buffer (50 mM Hepes, $\mathrm{pH} 7.9$, $150 \mathrm{mM} \mathrm{NaCl}, 0.5 \mathrm{mM}$ EDTA, $10 \%$ glycerol, $0.1 \%$ Triton X-100), we incubated the beads with HeLa nuclear extract $(40 \mu \mathrm{g})$ in binding buffer for $2 \mathrm{~h}$ at $4{ }^{\circ} \mathrm{C}$. Aliquots of the supernatant were then used for bandshift and western-blot analysis. We performed immunoprecipitations as described ${ }^{6}$. For immunoprecipitation of histone deacetylase activity ${ }^{6}$, we used HeLa whole cell extract, lysed in Tris (50 mM, pH 8.0), NaCl (150 mM), EDTA (0.5 $\mathrm{mM})$, EGTA $(0.5 \mathrm{mM}), 1 \% \mathrm{NP} 40, \beta$-mercaptoethanol $(5 \mathrm{mM})$ and protease inhibitors, as input.

Antibodies, western blots and affinity purification. Antibody S923 was raised in sheep against GST-MBD2a fusion protein. In this preparation of affinity-purified recombinant protein, only $20 \%$ was full length, so the sample was biased toward the tagged amino-terminal portion of MBD2a. Antibody R593 was raised in rabbit against GST-MBD2b fusion protein. Anti-mSin3A (AK11), anti-HDAC2 (H-52) and anti-Rb (C-15) were purchased (Santa Cruz). Anti-RbAp48 was raised against the synthetic peptide CENIYNDEDPEGSVDPEGQGS as described ${ }^{24}$. Antibodies against MeCP2 (ref. 5) have been described. We performed western blots as described $^{3}$, except the buffer used was Tris $\mathrm{HCl}(50 \mathrm{mM}, \mathrm{pH} 8.0), \mathrm{NaCl}$ $(150 \mathrm{mM}), 0.05 \%$ Tween-20 and $1 \%$ dried milk powder. Complexes were affinity purified using anti-HDACl antibodies as described ${ }^{11}$.

Demethylase assays. We carried out in vitro transcription/translation of MBD2 expression plasmids and demethylase assays as described ${ }^{8}$. We analysed restriction digests of MeCG11 probe by electrophoresis on $8 \%$ polyacrylamide gels.

\section{Acknowledgements}

We thank J. Millbrandt, M. Szyf, S. MacNeill and X. Nan for plasmids and J. Davidson and A. Greig for technical assistance. This work was supported by grants from the Wellcome Trust to A.B. and B.M.T. H.-H.N. holds a Darwin Trust Scholarship. D.R. is supported by the Howard Hughes Medical Institute and the National Institutes of Health. Y.Z. holds an NIH Post-doctoral Fellowship.

Received 3 May; accepted 1 July 1999.

3. Boyes, J. \& Bird, A. Repression of genes by DNA methylation depends on CPG 3oyes, J. \& Bird, A. Repression of genes by DNA methylation depends on CpG
density and promoter strength: evidence for involvement of a methyl-CpG binding protein. EMBO J. 11, 327-333 (1992).

14. Cameron, E.E., Bachman, K.E., Myohanen, S., Herman, J.G. \& Baylin, S.B. Synergy of demethylation and histone deacetylase inhilbition in the re-expression of genes silenced in cancer. Nature Genet. 21, 103-107 (1999).

15. Eden, S., Hashimshony, T., Keshet, I. \& Cedar, H. DNA methylation models histone acetylation. Nature 394, 842 (1998).

16. Ng, H.-H. \& Bird, A. DNA methylation and chromatin modification. Curr. Opin Genet. Dev. 9, 158-163 (1999).

17. Selker, E.U. Trichostatin A causes selective loss of DNA methylation in Neurospora. Proc. Natl Acad. Sci. USA 95, 9430-9435 (1998).

18. Brehm, A. et al. Retinoblastoma protein recruits histone deacetylase to repress transcription. Nature 391, 597-600 (1998).

19. Magnaghi-Jaulin, L. et al. Retinoblastoma protein represses transcription by recruiting a histone deacetylase. Nature 391, 601-604 (1998).

20. Luo, R.X., Postigo, A.A. \& Dean, D.C. Rb interacts with histone deacetylase to repress transcription. Cell 92, 463-473 (1998).

21. Nan, X., Tate, P., Li, E. \& Bird, A.P. DNA Methylation specifies chromosomal localization of MeCP2. Mol. Cell. Biol. 16, 414-421 (1996).

22. Nan, X., Meehan, R.R. \& Bird, A. Dissection of the methyl-CpG binding domain from the chromosomal protein MeCP2. Nucleic Acids Res. 21, 4886-4892 (1993).

23. Yeung, K.C., Inostroza, J.A., Mermelstein, F.H., Kannabiran, C. \& Reinberg, D. Structure-function analysis of the TBP-binding protein Dr1 reveals a mechanism for repression of class II gene transcription. Genes Dev. 8, 2097-2109 (1994).

24. White, D.A., Belyaev, N.D. \& Turner, B.M. Preparation of site-specific antibodies to acetylated histones. Methods (in press). 